\title{
Solutions to Open Problems on Fuzzy Filters of $B L$-algebras
}

\author{
Wang Wei ${ }^{1,2}$, Arsham Borumand Saeid ${ }^{3}$ \\ ${ }^{1}$ College of Electrical Engineering, Southwest Jiaotong University, \\ Chengdu, Sichuan, 610031, China \\ E-mail:wwmath@xsyu.edu.cn \\ ${ }^{2}$ College of Sciences, Xi'an Shiyou University, \\ Xi'an, Shaanxi, 710065, China \\ ${ }^{3}$ Department of Mathematics, Shahid Bahonar University of Kerman, \\ Kerman, Iran
}

Received 11 January 2014

Accepted 23 June 2014

\begin{abstract}
This paper focuses on the investigation of fuzzy filters of $B L$-algebras, an important and popular generic logical algebra. By studying the equivalent conditions of fuzzy fantastic filter and fuzzy normal filter of $B L$-algebras, the relation between these two filters are revealed. Two open problems of $B L$-algebras, i.e.,"Under what suitable condition a normal filter becomes a fantastic filter? " and "Under what suitable condition extension property for normal filter holds? (Extension property for a normal filter)", are solved accordingly.
\end{abstract}

Keywords: Non-classical logics; Artificial intelligence; $B L$-algebras; Fuzzy filter; Fuzzy fantastic filter; Fuzzy normal filter

\section{Introduction}

Logic algebras and the concept of filters play a vital role in reasoning mechanism in logic-based reasoning systems. Logic algebras are the algebraic foundation of the above systems and are the algebraic counterpart of the logic system. Hájek introduced $B L$-algebras as algebraic structures for his $\mathrm{Ba}-$ sic Logic $\left[^{1,2}\right]$. The main example of a $B L$-algebra is the interval $[0,1]$ endowed with the structure induced by a continuous t-norm. $M V$-algebras, Gödel algebras and product algebras are the most known classes of $B L$-algebras [3]

\footnotetext{
*corresponding author.
}

From the logical point of view, various filters correspond to various sets of provable formulae $\left[{ }^{4}\right]$. In $\left[{ }^{3,5,6}\right]$, the notions of prime filter, Boolean filter, implicative filters, normal filters, fantastic filters and positive implicative filters in $B L$-algebras were proposed, along with their properties investigated. In $\left[{ }^{7}\right]$, several different filters of residuated lattices and triangle-norm algebras were defined and their mutual dependencies and connections were examined. In [ $\left.{ }^{8,9,10}\right]$, filters of pseudo $M V$-algebras, pseudo $B L$ algebras, pseudo effect algebras and pseudo hoops were further studied.

At present, fuzzification ideas play an important 
role in the study of an algebraic structure $\left[{ }^{11}\right] . \mathrm{Xu}$ and Qin $\left[{ }^{12}\right]$ proposed the notions of fuzzy positive implicative filters in lattice implication algebras. Jun et al. derived several characterizations of fuzzy positive implicative filters of lattice implication algebras $\left[{ }^{13,14}\right]$. Some kinds of fuzzy filters of $B L$ algebras were further studied $\left[{ }^{5,3,15}\right]$. Wang and Xin solved an open problem in pseudo $B L$-algebras by discussing the relation between fuzzy normal and fuzzy Boolean filter in $\left[{ }^{16}\right]$. In $\left[{ }^{17,18,19}\right]$, interval valued $(\in, \in \vee q)$-fuzzy filters of residuated lattice and $M T L$-algebras were studied. Fuzzy filters are not only an extension of classical filter, but also a useful tool to infer some important results on classical filters due to the investigation from different point of views.

In Section 2, some basic definitions and results on fuzzy filters and $B L$-algebras are reviewed. In Section 3, some kinds of fuzzy filters in $B L$-algebras are introduced.

In $\left[{ }^{6}\right]$, Saeid and Motamed proposed two open problems that "Under what suitable condition a normal filter becomes a fantastic filter? " and "(Extension property for a normal filter) Under what suitable condition extension property for normal filter holds? "in $B L$-algebras. In Section 4 , by studying the equivalent condition of fuzzy fantastic filter and fuzzy normal filter, we reveal the relation between fuzzy fantastic filter and fuzzy normal filter of $B L$ algebras and solved these two open problems.

\section{Preliminaries}

Here we recall some definitions and results which will be needed.

Definition 1. (Hájek $\left.\left[{ }^{1}\right]\right)$ A $B L$-algebra is an algebra $(A, \vee, \wedge, \odot, \rightarrow, 0,1)$ of type $(2,2,2,2,2,0,0)$ such that $(A, \vee, \wedge, 0,1)$ is a bounded lattice, $(A, \odot, 1)$ is a commutative monoid and the following conditions hold for all $x, y, z \in A$

(A1) $x \odot y \leqslant z$ if and only if $x \leqslant y \rightarrow z$,

(A2) $x \wedge y=x \odot(x \rightarrow y)$,

(A3) $(x \rightarrow y) \vee(y \rightarrow x)=1$.

Proposition 1. (Liu and Li $\left[^{3}\right]$ ) In a BL-algebra A, the following properties hold for all $x, y, z \in A$

$$
\text { (1) } y \rightarrow(x \rightarrow z)=x \rightarrow(y \rightarrow z) \text {, }
$$

(2) $1 \rightarrow x=x$,

(3) $x \leqslant y$ iff $x \rightarrow y=1$,

(4) $x \vee y=((x \rightarrow y) \rightarrow y) \wedge((y \rightarrow x) \rightarrow x)$,

(5) $x \leqslant y \Rightarrow y \rightarrow z \leqslant x \rightarrow z$

(6) $x \leqslant y \Rightarrow z \rightarrow x \leqslant z \rightarrow y$,

(7) $x \rightarrow y \leqslant(z \rightarrow x) \rightarrow(z \rightarrow y)$,

(8) $x \rightarrow y \leqslant(y \rightarrow z) \rightarrow(x \rightarrow z)$,

(9) $x \leqslant(x \rightarrow y) \rightarrow y$,

$(10) x \odot(x \rightarrow y)=x \wedge y$.

In the sequel, we shall use $A$ to denote a $B L$ algebras and agree that the operation $\vee, \wedge, \odot$ have priority towards the operations $\rightarrow$.

In a $B L$-algebra $A$, we can define: $x^{-}=x \rightarrow 0$ for any $x \in A$.

$M V$-algebras and $B L$-algebras are closely related. Indeed, a $B L$-algebra $A$ is an $M V$-algebra iff $x^{--}=x$ for all $x \in A\left[{ }^{20}\right]$.

Here we recall some kinds of filters and fuzzy filters in $B L$-algebras.

Definition 2. (Zhang $\left[{ }^{21}\right]$ ) A filter of a $B L$-algebra A is a nonempty subset $\mathrm{F}$ of $\mathrm{A}$ such that for all $x, y \in A$

(F1) if $x, y \in F$, then $x \odot y \in F$,

(F2) if $x \in F$ and $x \leqslant y$, then $y \in F$.

It is easy to prove the following equivalent conditions of filter in a $B L$-algebra.

Proposition 2. (Zhang $\left.\left[{ }^{21}\right]\right)$ Let $F$ be a nonempty subset of a BL-algebra A. Then $F$ is a filter of $A$ if and only if the following conditions hold

(1) $1 \in F$,

(2) $x, x \rightarrow y \in F$ implies $y \in F$.

A filter $F$ of a $B L$-algebra $A$ is proper if $F \neq A$.

Definition 3. (Borumand $\left[{ }^{6}\right]$ ) A filter $F$ of $A$ is called normal if for any $x, y, z \in A, z \rightarrow((y \rightarrow x) \rightarrow x) \in F$ and $z \in F$ imply $(x \rightarrow y) \rightarrow y \in F$.

Definition 4. (Borumand $\left[{ }^{6}\right]$ )Let $F$ be a nonempty subset of a $B L$-algebra $\mathrm{A}$. Then $\mathrm{F}$ is called a fantastic filter of $\mathrm{A}$ if for all $x, y, z \in A$, the following conditions hold

(1) $1 \in F$,

(2) $z \rightarrow(y \rightarrow x) \in F, z \in F$ implies $((x \rightarrow y) \rightarrow$ $y) \rightarrow x \in F$.

Definition 5. (Borumand $\left[{ }^{6}\right]$ )Let $F$ be a nonempty subset of a $B L$-algebra $\mathrm{A}$. Then $\mathrm{F}$ is called an implicative filter of $\mathrm{A}$ if for all $x, y, z \in A$, the following conditions hold 
(1) $1 \in F$,

(2) $x \rightarrow(y \rightarrow z) \in F, x \rightarrow y \in F$ imply $x \rightarrow z \in F$.

Definition 6. (Borumand $\left[{ }^{6}\right]$ )Let $\mathrm{F}$ be a nonempty subset of a $B L$-algebra $\mathrm{A}$. Then $\mathrm{F}$ is called a positive implicative filter of $\mathrm{A}$ if for all $x, y, z \in A$, the following conditions hold

(1) $1 \in F$,

(2) $x \rightarrow((y \rightarrow z) \rightarrow y) \in F, x \in F$ imply $y \in F$.

Definition 7. (Borumand $\left[{ }^{6}\right]$ )A filter $\mathrm{F}$ of $\mathrm{A}$ is called Boolean if $x \vee x^{-} \in F$ for any $x \in A$,

Definition 8. (Zhang $\left[{ }^{21}\right]$ ) Let $\mathrm{F}$ be a filter of A. F is called an ultra filter of $\mathrm{A}$ if it satisfies $x \in F$ or $x^{-} \in F$ for all $x \in A$.

Definition 9. (Zhang $\left[{ }^{21}\right]$ ) Let $\mathrm{F}$ be a filter of A. $\mathrm{F}$ is called an obstinate filter of A if it satisfies $x \notin F$ and $y \notin F$ implies $x \rightarrow y \in F$ for all $x, y \in A$.

Definition 10. (Liu and $\left.\mathrm{Li}^{3}\right]$ )A fuzzy filter of a $B L-$ algebra $\mathrm{A}$ is a fuzzy subset $\mathrm{f}$ of $\mathrm{A}$ such that for all $x, y \in A$

(1) $f(1) \geqslant f(x)$

(2) $f(y) \geqslant f(x) \wedge f(x \rightarrow y)$.

Proposition 3. (Liu and $\left.\mathrm{Li}^{3}\right]$ ) Let $f$ be a fuzzy set of a BL-algebra $A$. $f$ is a fuzzy filter of $A$ if and only if for all $x, y, z \in A, x \rightarrow(y \rightarrow z)=1$ implies $f(z) \geqslant f(x) \wedge f(y)$.

Corollary 4. (Liu and Li $\left.{ }^{3}\right]$ ) Let $f$ be a fuzzy set of a $B L$-algebra $A$. $f$ is a fuzzy filter of $A$ if and only if for all $x, y, z \in A, x \odot y \leqslant z$ implies $f(z) \geqslant f(x) \wedge f(y)$.

Proposition 5. (Liu and $\mathrm{Li}\left[{ }^{3}\right]$ ) Let f be a fuzzy set of a BL-algebra A. $f$ is a fuzzy filter of $A$ if and only if

(1) $f$ is order-preserving,

(2) $f(x \odot y) \geqslant f(x) \wedge f(y)$ for any $x, y \in A$.

Corollary 6. (Liu and Li[ $\left.{ }^{3}\right]$ ) Let $f$ be an orderpreserving fuzzy set of a BL-algebra $A$. $f$ is a fuzzy filter of $A$ if and only if $f(x \odot y)=f(x) \wedge f(y)$ for any $x, y \in A$.

We can easily get the following corollaries.

Corollary 7. Let $f$ be a fuzzy filter of a BL-algebra $A$, then for any $x \in A, f(x)=f(x \odot x)=f(x \odot x \odot$ $\ldots \odot x)$.
Corollary 8. (Liu and Li $\left[^{3}\right]$ ) Let $f$ be a fuzzy filter of a BL-algebra $A$, then $f(x \wedge y)=f(x) \wedge f(y)$ for any $x, y \in A$.

Lemma 9. Let $f$ be a fuzzy filter in a BL algebra $A$. For any $x, y \in A$, if $f(x \rightarrow y)=f(1)$, then $f(x) \leqslant f(y)$.

Proof. Let $f(x)=t$, then $x \in f_{t}$. if $f(x \rightarrow y)=f(1)$, then $x \rightarrow y \in f_{f(1)}$, it follows that $x \rightarrow y \in f_{t} . f_{t}$ is a filter, then we have $y \in f_{t}$. This implies that $f(y) \geqslant t=f(x)$. Dually, if $f(x \hookrightarrow y)=f(1)$, then $f(x) \leqslant f(y)$.

\section{Some kinds of fuzzy filters of $B L$-algebras}

To discuss the relation among the filters of a $B L$ algebra, we establish the fuzzification of them and obtain some important properies.

Definition 11. Let A be a $B L$-algebra. A fuzzy filter $\mathrm{f}$ of a $B L$-algebra $\mathrm{A}$ is called fuzzy normal if for any $x, y, z \in A$,

$$
f((x \rightarrow y) \rightarrow y) \geqslant f(z \rightarrow((y \rightarrow x) \rightarrow x)) \wedge f(z) .
$$

Definition 12. Let $\mathrm{f}$ be a fuzzy subset of a $B L$ algebra $\mathrm{A}$. Then $\mathrm{f}$ is called a fuzzy fantastic filter of A if for all $x, y, z \in A$, the following conditions hold

(1) $f(1) \geqslant f(x)$, $f(z)$.

(2) $f(((x \rightarrow y) \rightarrow y) \rightarrow x) \geqslant f(z \rightarrow(y \rightarrow x)) \wedge$

Definition 13. Let $f$ be a fuzzy subset of a $B L$ algebra $\mathrm{A}$. Then $\mathrm{f}$ is called a fuzzy implicative filter of $\mathrm{A}$ if for all $x, y, z \in A$, the following conditions hold

(1) $f(1) \geqslant f(x)$,

(2) $f(x \rightarrow z) \geqslant f(x \rightarrow(y \rightarrow z)) \wedge f(x \rightarrow y)$.

Definition 14. Let $f$ be a fuzzy subset of a $B L-$ algebra $\mathrm{A}$. Then $\mathrm{f}$ is called a fuzzy positive implicative filter of $\mathrm{A}$ if for all $x, y, z \in A$, the following conditions hold

(1) $f(1) \geqslant f(x)$,

(2) $f(y) \geqslant f(x \rightarrow((y \rightarrow z) \rightarrow y)) \wedge f(x)$.

Definition 15. (Liu and Li $\left[{ }^{5}\right]$ )Let $f$ be a fuzzy filter of A. Then $\mathrm{f}$ is a fuzzy Boolean filter of $\mathrm{A}$, if for all $x \in A, f\left(x \vee x^{-}\right)=f(1)$. 
Definition 16. (Zhang, Jun and Doh $\left[{ }^{15}\right]$ )A fuzzy set $\mathrm{f}$ of $\mathrm{A}$ is called a fuzzy ultra filter of $\mathrm{A}$ if for all $x \in A$ it is a fuzzy filter of A that satisfies the following conditions

$$
f(x)=f(1) \text { or } f\left(x^{-}\right)=f(1) \text { for all } x \in A .
$$

Definition 17. (Zhang, Jun and Doh $\left[{ }^{15}\right]$ )A fuzzy set $\mathrm{f}$ of $\mathrm{A}$ is called a fuzzy obstinate filter of $\mathrm{A}$ if it is a fuzzy filter of A that satisfies the following conditions

$f(x) \neq f(1)$ and $f(y) \neq f(1)$ imply $f(x \rightarrow y)=f(1)$ and $f(y \rightarrow x)=f(1)$

for all $x, y \in A$.

Theorem 10. (Zhang, Jun and Doh $\left[{ }^{15}\right]$ )Every fuzzy obstinate filter of a BL-algebra is equivalent to a fuzzy ultra filter.

Theorem 11. Let $f$ be a fuzzy filter of a BL-algebra A. Then $f$ is a fuzzy normal(fantastic,(positive)implicative, Boolean,obstinate, ultra) filter of $A$ if and only if for each $t \in[0,1], f_{t}$ is either empty or a normal(fantastic,(positive) implicative, Boolean,obstinate, ultra) filter of A.

Proof. Suppose $f$ is a fuzzy normal filter of $A$. For any $x, y, z \in A$, if $f_{t} \neq \varnothing$, and $z, z \rightarrow((y \rightarrow x) \rightarrow$ $x) \in f_{t}$, then $f((x \rightarrow y) \rightarrow y) \geqslant f(z \rightarrow((y \rightarrow x) \rightarrow$ $x) \wedge f(z) \geqslant t$, we have $(x \rightarrow y) \rightarrow y \in f_{t}$, i.e. $f_{t}$ is a normal filter.

Conversely, suppose that for each $t \in[0,1], f_{t}$ is either empty or a normal filter. Let $t=f(z \rightarrow((y \rightarrow$ $x) \rightarrow x)) \wedge f(z)$, then $z \rightarrow((y \rightarrow x) \rightarrow x), z \in f_{t}$. This implies that $f_{t}$ is a normal filter. So $(x \rightarrow y) \rightarrow y \in f_{t}$, i.e., $f((x \rightarrow y) \rightarrow y) \geqslant f(z \rightarrow((y \rightarrow x) \rightarrow x)) \wedge f(z)$ and hence $f$ is a fuzzy normal filter.

In the same way, we can get the other results.

Theorem 12. Let $f$ be a fuzzy filter of a BL-algebra A. $f$ is a fuzzy normal(fantastic,(positive)implicative, Boolean,obstinate, ultra) filter of $A$ if and only if $f_{f(1)}$ is a normal(fantastic,(positive)implicative, Boolean,obstinate, ultra) filter of $A$.

Proof. Necessity is obvious. Suppose $f_{f(1)}$ is a normal filter, for any $t \in[0,1]$, if $f_{t} \neq \phi$, suppose $z \rightarrow$ $((y \rightarrow x) \rightarrow x), z \in f_{f(1)}$, then $(x \rightarrow y) \rightarrow y \in f_{f(1)}$, $f(z \rightarrow((y \rightarrow x) \rightarrow x))=f(1)$ and $f(z)=f(1)$, so $f((x \rightarrow y) \rightarrow y)=f(1) \geqslant f(z \rightarrow((y \rightarrow x) \rightarrow$ $x)) \wedge f(z)$, thus $f$ is a fuzzy normal filter of $A$. In the similar way, we get the other results.

Corollary 13. Let $F$ be a nonempty subset of a BL-algebra A. $F$ is a normal(fantastic,(positive)implicative,Boolean,obstinate, ultra) filter of $A$ if and only if $\chi_{F}$ is a fuzzy normal(fantastic,(positive)implicative, Boolean,obstinate, ultra) filter of $A$ where $\chi_{F}$ is the characteristic function of $F$.

\section{The relation among the fuzzy filters of $B L$-algebras}

In $\left[{ }^{6}\right]$, there are two open problems, i.e., 1) "Under what suitable condition a normal filter becomes a fantastic filter?" and 2) "(Extension property for a normal filter) Under what suitable condition extension property for normal filter holds? "To solve the open problems, it is very useful to discuss the relation between fuzzy fantastic filter and fuzzy normal filter of $B L$-algebras. In this section, we investigate the relation among these two filters of a $B L$-algebras. After giving the equivalent conditions of these two fuzzy filters, we present the relation between fuzzy fantastic filter and fuzzy normal filter of $B L$-algebras and solve the two open problems.

Theorem 14. Let $f$ be a fuzzy filter of a BL-algebra A. $f$ is a fuzzy normal filter if only if $f((y \rightarrow x) \rightarrow$ $x)=f((x \rightarrow y) \rightarrow y)$ for all $x, y \in A$.

Proof. Let $f$ be an fuzzy normal filter of $A$ and let $f((y \rightarrow x) \rightarrow x)=t$. Then $(y \rightarrow x) \rightarrow x \in f_{t}$. Since $1 \rightarrow((y \rightarrow x) \rightarrow x)=(y \rightarrow x) \rightarrow x \in f_{t}, 1 \in f_{t}$, and $f_{t}$ is a normal filter, thus $\left.(x \rightarrow y) \rightarrow y\right) \in f_{t}$, so $f((x \rightarrow y) \rightarrow y) \geqslant t=f((y \rightarrow x) \rightarrow x)$. Dually we can get $f((x \rightarrow y) \rightarrow y) \leqslant f((y \rightarrow x) \rightarrow x)$.

Conversely, let $f(z \rightarrow((y \rightarrow x) \rightarrow x)) \wedge f(z)=t$, then $z \rightarrow((y \rightarrow x) \rightarrow x), z \in f_{t}$. Since $f$ is a filter, then $(y \rightarrow x) \rightarrow x \in f_{t}$, then we get that $(x \rightarrow y) \rightarrow$ $y \in f_{t}$, thus $f((x \rightarrow y) \rightarrow y) \geqslant t=f(z \rightarrow((y \rightarrow x) \rightarrow$ $x)) \wedge f(z)$. Hence $f$ is a normal filter of $A$.

Theorem 15. Let $f$ be a fuzzy filter of a BL-algebra A. $f$ is a fuzzy normal filter if only if $f\left(x^{--}\right)=f(x)$ for all $x \in A$. 
Proof. Necessity is obvious when $y=0$ in the above theorem.

Conversely, for all $x, y \in A$, let $f((x \rightarrow y) \rightarrow y)=$ $t$, then $(x \rightarrow y) \rightarrow y \in f_{t}$. When $y=0$, then $x^{--} \in f_{t}$, so we get that $x \in f_{t}$, thus $x \leqslant(y \rightarrow x) \rightarrow x \in f_{t}$. Then $f((y \rightarrow x) \rightarrow x) \geqslant t=f((x \rightarrow y) \rightarrow y)$. Dually we can get $f((y \rightarrow x) \rightarrow x) \leqslant f((x \rightarrow y) \rightarrow y)$. Hence $f$ is a fuzzy normal filter of $A$.

Theorem 16. Let $f$ be a fuzzy implicative filter of a $B L$-algebra $A$. Then $f$ is a normal filter if and only if $f((x \rightarrow y) \rightarrow x)=f(x)$ for any $x, y \in A$.

Proof. Suppose $f$ is a fuzzy implicative filter of a $B L$-algebra $A$ satisfying $f((x \rightarrow y) \rightarrow x)=f(x)$ for any $x, y \in A$. Let $f((x \rightarrow y) \rightarrow y)=t$. Then we have $(x \rightarrow y) \rightarrow y \leqslant(y \rightarrow x) \rightarrow((x \rightarrow y) \rightarrow$ $x)=(x \rightarrow y) \rightarrow((y \rightarrow x) \rightarrow x)$. Since $F$ is a filter, then $(x \rightarrow y) \rightarrow((y \rightarrow x) \rightarrow x) \in f_{t}$. And we have $x \leqslant(y \rightarrow x) \rightarrow x$, so $((y \rightarrow x) \rightarrow x) \rightarrow y \leqslant$ $x \rightarrow y$, thus $(x \rightarrow y) \rightarrow((y \rightarrow x) \rightarrow x) \leqslant(((y \rightarrow$ $x) \rightarrow x) \rightarrow y) \rightarrow((y \rightarrow x) \rightarrow x)$. Then we get that $(((y \rightarrow x) \rightarrow x) \rightarrow y) \rightarrow((y \rightarrow x) \rightarrow x) \in f_{t}$, then we can conclude $(y \rightarrow x) \rightarrow x \in f_{t}$, i.e. $f((y \rightarrow$ $x) \rightarrow x) \geqslant t=f((x \rightarrow y) \rightarrow y)$. Dually we can get $f((y \rightarrow x) \rightarrow x) \leqslant f((x \rightarrow y) \rightarrow y)$. Therefore $f$ is a fuzzy normal filter.

Conversely, let $f((x \rightarrow y) \rightarrow x)=t$, then $(x \rightarrow$ $y) \rightarrow x \in f_{t}$. By Corollary 2 of $\left[{ }^{6}\right]$, we get that $f((x \rightarrow y) \rightarrow x) \leqslant f(x)$. Since $(x \rightarrow y) \rightarrow x \geqslant x$ and $f$ is isotone, we can easily get $f((x \rightarrow y) \rightarrow x)=f(x)$.

Corollary 17. Let $f$ be a fuzzy filter of A satisfying $f((x \rightarrow y) \rightarrow x)=f(x)$ for any $x, y \in A$. Then $f$ is a fuzzy normal filter.

Corollary 18. Let $f$ be a fuzzy filter of A satisfying $f\left(x^{-} \rightarrow x\right)=f(x)$ for any $x \in A$. Then $f$ is a fuzzy normal filter.

According to the above theorem and corollaries we have

Theorem 19. Let $f$ be a fuzzy implicative filter of $A$. Then for any $x, y \in A$, the following conditions are equivalent:

(1) $f$ is a fuzzy normal filter,

(2) $f$ is a fuzzy filter of A satisfying $f((x \rightarrow y) \rightarrow$ $x)=f(x)$, $f(x)$.

(3) $f$ is a fuzzy filter of $A$ satisfying $f\left(x^{-} \rightarrow x\right)=$

Theorem 20. (Liu and Li $\left[^{5}\right]$ )Let $f$ be a fuzzy filter of $A$. The following are equivalent:

(1) $f$ is a fuzzy Boolean filter of $A$,

(2) $f((x \rightarrow y) \rightarrow x)=f(x)$ for all $x, y \in A$,

(3) $f\left(x^{-} \rightarrow x\right)=f(x)$ for all $x \in A$.

Theorem 21. Let $f$ be a fuzzy Boolean filter of A, then for all $x, y \in A$

(1) $f(((y \rightarrow x) \rightarrow x) \rightarrow y)=f(x \rightarrow y)$,

(2) $f\left(\left(x \rightarrow x^{-}\right)=f\left(x^{-}\right)\right.$

(3) $f\left(x^{--}\right)=f(x)$.

Proof. (1)Assume $f(x \rightarrow y)=t$, then $x \rightarrow y \in f_{t}$. From $y \rightarrow x \leqslant((y \rightarrow x) \rightarrow x) \rightarrow x$, we get $(y \rightarrow$ $x) \rightarrow(((y \rightarrow x) \rightarrow x) \rightarrow y) \geqslant(((y \rightarrow x) \rightarrow x) \rightarrow x) \rightarrow$ $(((y \rightarrow x) \rightarrow x) \rightarrow y) \geqslant x \rightarrow y \in f_{t}$. That is, $(y \rightarrow$ $x) \rightarrow(((y \rightarrow x) \rightarrow x) \rightarrow y) \in f_{t}$. And, $y \leqslant((y \rightarrow$ $x) \rightarrow x) \rightarrow y, y \rightarrow x \geqslant(((y \rightarrow x) \rightarrow x) \rightarrow y) \rightarrow x$. We can get $((((y \rightarrow x) \rightarrow x) \rightarrow y) \rightarrow x) \rightarrow(((y \rightarrow x) \rightarrow$ $x) \rightarrow y) \geqslant(y \rightarrow x) \rightarrow(((y \rightarrow x) \rightarrow x) \rightarrow y) \in f_{t}$. That is, $((((y \rightarrow x) \rightarrow x) \rightarrow y) \rightarrow x) \rightarrow(((y \rightarrow x) \rightarrow x) \rightarrow$ $y) \in f_{t}$. Using the above Theorem, we have $((y \rightarrow$ $x) \rightarrow x) \rightarrow y \in f_{t}$. i.e., $f(((y \rightarrow x) \rightarrow x) \rightarrow y) \geqslant t$. It is easy to see $f(((y \rightarrow x) \rightarrow x) \rightarrow y) \leqslant t$, then we get $f(((y \rightarrow x) \rightarrow x) \rightarrow y)=t$.

(2) From $f\left(x \vee x^{-}\right)=f(1)=f\left(\left(\left(x \rightarrow x^{-}\right) \rightarrow\right.\right.$ $\left.\left.x^{-}\right) \wedge\left(\left(x^{-} \rightarrow x\right) \rightarrow x\right)\right)=f\left(\left(x \rightarrow x^{-}\right) \rightarrow x^{-}\right) \wedge$ $f\left(\left(x^{-} \rightarrow x\right) \rightarrow x\right)$, we get $f\left(\left(x \rightarrow x^{-}\right) \rightarrow x^{-}\right)=f(1)$, and by Lemma, we get $f\left(x \rightarrow x^{-}\right)=f\left(x^{-}\right)$.

(3) Let $x=0$ in (1) and by Lemma 9, we can get the result.

Corollary 22. Each fuzzy Boolean filter of $A$ is a fuzzy normal filter.

Theorem 23. Let $f$ be a fuzzy filter of a BL-algebra $A$. Then the followings are equivalent:

(1) $f$ is a fantastic filter,

(2) $f(y \rightarrow x)=f(((x \rightarrow y) \rightarrow y) \rightarrow x)$ for any $x, y \in A$,

(3) $f\left(x^{--} \rightarrow x\right)=f(1)$, for all $x \in A$,

(4) $f(((x \rightarrow y) \rightarrow y) \rightarrow z) \geqslant f(x \rightarrow z) \wedge f(y \rightarrow z)$ for all $x, y, z \in A$.

Proof. $(1) \Rightarrow(2)$. Let $f$ be a fuzzy fantastic filter of $A$ and $f(y \rightarrow x)=t$. Then $y \rightarrow x \in f_{t}$. Then $1 \rightarrow(y \rightarrow x)=y \rightarrow x \in f_{t}$ and $1 \in f_{t}$, hence $((x \rightarrow$ 
$y) \rightarrow y) \rightarrow x \in f_{t}$, i.e., $f(((x \rightarrow y) \rightarrow y) \rightarrow x) \geqslant t=$ $f(y \rightarrow x)$. Since $((x \rightarrow y) \rightarrow y) \rightarrow x \leqslant y \rightarrow x$, we can get $f(((x \rightarrow y) \rightarrow y) \rightarrow x) \leqslant f(y \rightarrow x)$. Thus $f(((x \rightarrow y) \rightarrow y) \rightarrow x)=f(y \rightarrow x)$.

$(2) \Rightarrow(1)$. let a fuzzy filter $f$ satisfy the condition and let $f(z \rightarrow(y \rightarrow x)) \wedge f(z)=t$, then $z \rightarrow$ $(y \rightarrow x) \in f_{t}$ and $z \in f_{t}$. Then $y \rightarrow x \in f_{t}$, therefore $f(((x \rightarrow y) \rightarrow y) \rightarrow x) \geqslant t$, i.e., $((x \rightarrow y) \rightarrow y) \rightarrow x \in$ $f_{t}$. Thus $f$ is a fantastic filter.

$(2) \Rightarrow(3)$. Let $y=0$ in (2).

(3) $\Rightarrow(4)$. Let $f(x \rightarrow z) \wedge f(y \rightarrow z)=t$ for all $x, y, z \in A$, then $x \rightarrow z, y \rightarrow z \in f_{t}$. Since $x \rightarrow z \leqslant$ $z^{-} \rightarrow x^{-} \in f_{t}, y \rightarrow z \leqslant z^{-} \rightarrow y^{-} \in f_{t}$, so $\left(z^{-} \rightarrow\right.$ $\left.x^{-}\right) \wedge\left(z^{-} \rightarrow y^{-}\right) \in f_{t}$. And $\left(z^{-} \rightarrow x^{-}\right) \wedge\left(z^{-} \rightarrow y^{-}\right)=$ $z^{-} \rightarrow\left(x^{-} \wedge y^{-}\right)=z^{-} \rightarrow y^{-} \odot\left(y^{-} \rightarrow x^{-}\right)=z^{-} \rightarrow$ $y^{-} \odot\left(y^{-} \rightarrow(x \rightarrow 0)\right)=z^{-} \rightarrow y^{-} \odot\left(x \rightarrow\left(y^{-} \rightarrow\right.\right.$ $0))=z^{-} \rightarrow y^{-} \odot\left(x \rightarrow y^{--}\right)$.

And $\left(z^{-} \rightarrow y^{-} \odot\left(x \rightarrow y^{--}\right)\right) \rightarrow\left(z^{-} \rightarrow y^{-} \odot(x \rightarrow\right.$ $y)) \geqslant\left(y^{-} \odot\left(x \rightarrow y^{--}\right)\right) \rightarrow\left(y^{-} \odot(x \rightarrow y)\right) \geqslant(x \rightarrow$ $\left.y^{--}\right) \rightarrow(x \rightarrow y) \geqslant y^{--} \rightarrow y \in f_{t}$, so $z^{-} \rightarrow y^{-} \odot(x \rightarrow$ $y) \in f_{t}$. And $z^{-} \rightarrow y^{-} \odot(x \rightarrow y) \leqslant\left(y^{-} \odot(x \rightarrow\right.$ $y))^{-} \rightarrow z^{--}=\left((x \rightarrow y) \rightarrow y^{--}\right) \rightarrow z^{--} \in f_{t}$. Further $\left(\left((x \rightarrow y) \rightarrow y^{--}\right) \rightarrow z^{--}\right) \rightarrow(((x \rightarrow y) \rightarrow y) \rightarrow$ $\left.z^{--}\right) \geqslant((x \rightarrow y) \rightarrow y) \rightarrow\left((x \rightarrow y) \rightarrow y^{--}\right) \geqslant y \rightarrow$ $y^{--}=1 \in f_{t}$, so $((x \rightarrow y) \rightarrow y) \rightarrow z^{--} \in f_{t}$. And $\left(((x \rightarrow y) \rightarrow y) \rightarrow z^{--}\right) \rightarrow(((x \rightarrow y) \rightarrow y) \rightarrow z) \geqslant$ $z^{--} \rightarrow z \in f_{t}$, so $((x \rightarrow y) \rightarrow y) \rightarrow z \in f_{t}$. Then $f(((x \rightarrow y) \rightarrow y) \rightarrow z) \geqslant t=f(x \rightarrow z) \wedge f(y \rightarrow z)$.

(4) $\Rightarrow(2)$. Obvious when $z=x$.

Corollary 24. Each fuzzy fantastic filter of $A$ is a fuzzy normal filter.

Corollary 25. Each fuzzy normal filter of $A$ is a fuzzy fantastic filter if the inverse condition of Lemma 9 holds.

Proof. For any fuzzy filter $f$ of $A, f$ is a fuzzy normal filter if and only if $f\left(x^{--}\right)=f(x)$ for all $x \in A$ if and only if $f\left(x^{--}\right) \leqslant f(x)$ for all $x \in A$, then we get $f\left(x^{--} \rightarrow x\right)=f(1)$ for $x \in A$, i.e., $f$ is a fuzzy fantastic filter.

Theorem 26. Let $f$ be a fuzzy filter of a BL-algebra A. Then the followings are equivalent

(1) $f$ is a fuzzy implicative filter,

(2) $f(y \rightarrow x)=f(y \rightarrow(y \rightarrow x))$ for any $x, y \in A$,
(3) $f(y \rightarrow x) \geqslant f(z \rightarrow(y \rightarrow(y \rightarrow x))) \wedge f(z)$ for any $x, y, z \in A$,

(4) $f(x \rightarrow x \odot x)=f(1)$ for all $x \in A$.

Proof. $(1) \Rightarrow(2)$. Let $f$ be a fuzzy implicative filter of $A$ and $f(y \rightarrow(y \rightarrow x))=t$. Then $y \rightarrow(y \rightarrow$ $x) \in f_{t}$. And $y \rightarrow y=1 \in f_{t}$ hence $y \rightarrow x \in f_{t}$, i.e., $f(y \rightarrow x) \geqslant t=f(y \rightarrow(y \rightarrow x))$. The inverse inequation is obvious.

$(2) \Rightarrow(3)$. let $f$ be a fuzzy filter satisfying the condition and let $f(z \rightarrow(y \rightarrow(y \rightarrow x))) \wedge f(z)=t$, then $z \rightarrow(y \rightarrow(y \rightarrow x)) \in f_{t}$ and $z \in f_{t}$. Then $y \rightarrow$ $(y \rightarrow x) \in f_{t}$, therefore $f(y \rightarrow x)=f(y \rightarrow(y \rightarrow x)) \geqslant$ $t$, i.e., $f(y \rightarrow x) \geqslant f(z \rightarrow(y \rightarrow(y \rightarrow x))) \wedge f(z)$.

(3) $\Rightarrow(1)$. Let $f(x \rightarrow(y \rightarrow z)) \wedge f(x \rightarrow y)=t$ for all $x, y, z \in A$, then $x \rightarrow(y \rightarrow z), x \rightarrow y \in f_{t}$. Since $x \rightarrow(y \rightarrow z)=y \rightarrow(x \rightarrow z) \leqslant(x \rightarrow y) \rightarrow(x \rightarrow(x \rightarrow$ $z)) \in f_{t}$, so $x \rightarrow z \in f_{t}$ and $f$ is a fuzzy implicative filter.

$(1) \Rightarrow(4)$. Let $f$ be a fuzzy implicative filter of $A$. And for all $x \in A, f(x \rightarrow(x \rightarrow x \odot x))=f(x \odot x \rightarrow$ $x \odot x)=f(1), f(x \rightarrow x)=f(1)$, so $f(x \rightarrow x \odot x)=$ $f(1)$.

$(4) \Rightarrow(1)$. let a fuzzy filter $f$ satisfy the condition and let $f(x \rightarrow(y \rightarrow z)) \wedge f(x \rightarrow y)=t$, then $x \rightarrow(y \rightarrow z) \in f_{t}$ and $x \rightarrow y \in f_{t}$. Then $(x \rightarrow$ $(y \rightarrow z)) \odot(x \rightarrow y) \odot x \odot x \leqslant(y \rightarrow z) \odot y \leqslant z$, therefore $(x \rightarrow(y \rightarrow z)) \odot(x \rightarrow y) \leqslant x \odot x \rightarrow z \leqslant(x \rightarrow$ $x \odot x) \rightarrow(x \rightarrow z) \in f_{t}$, so $x \rightarrow z \in f_{t}$, i.e., $f(x \rightarrow z) \geqslant$ $t=f(x \rightarrow(y \rightarrow z)) \wedge f(x \rightarrow y)$, and $f$ is a fuzzy implicative filter.

Theorem 27. Let $f$ be a fuzzy filter of a BL-algebra $A$. Then the the following statements are equivalent:

(1) $f$ is a fuzzy positive implicative filter,

(2) $f(x)=f((x \rightarrow y) \rightarrow x)$ for any $x, y \in A$,

(3) $f\left(\left(x^{-} \rightarrow x\right) \rightarrow x\right)=f(1)$ for any $x \in A$.

Proof. $(1) \Rightarrow(2)$. Let $f$ be a fuzzy implicative filter of $A$ and $f((x \rightarrow y) \rightarrow x)=t$. Then $(x \rightarrow y) \rightarrow x \in f_{t}$. And $1 \rightarrow((x \rightarrow y) \rightarrow x)=(x \rightarrow y) \rightarrow x \in f_{t}$ and $1 \in f_{t}$, hence $x \in f_{t}$, i.e., $f(x) \geqslant t=f((x \rightarrow y) \rightarrow x)$. The inverse inequality is obvious.

$(2) \Rightarrow(1)$. let $f$ be a fuzzy filter satisfying the condition and let $f(x \rightarrow((y \rightarrow z) \rightarrow y)) \wedge f(x)=t$, then $x \rightarrow((y \rightarrow z) \rightarrow y) \in f_{t}$ and $x \in f_{t}$. Then $(y \rightarrow z) \rightarrow y \in f_{t}$, therefore $y \in f_{t}$, i.e., $f(y) \geqslant f(x \rightarrow$ $((y \rightarrow z) \rightarrow y)) \wedge f(x)$. 
(2) $\Rightarrow$ (3). For $\forall x \in A, f\left(\left(\left(\left(x^{-} \rightarrow x\right) \rightarrow x\right) \rightarrow\right.\right.$ $\left.0) \rightarrow\left(\left(x^{-} \rightarrow x\right) \rightarrow x\right)\right)=f\left(\left(x^{-} \rightarrow x\right) \rightarrow\left(\left(\left(\left(x^{-} \rightarrow\right.\right.\right.\right.\right.$ $x) \rightarrow x)) \rightarrow 0) \rightarrow x) \geqslant\left(\left(\left(x^{-} \rightarrow x\right) \rightarrow x\right) \rightarrow 0\right) \rightarrow$ $x^{-}=\left(\left(\left(x^{-} \rightarrow x\right) \rightarrow x\right) \rightarrow 0\right) \rightarrow(x \rightarrow 0) \geqslant x \rightarrow$ $\left(\left(x^{-} \rightarrow x\right) \rightarrow x\right)=f(1)$, then $f\left(\left(\left(\left(x^{-} \rightarrow x\right) \rightarrow x\right) \rightarrow\right.\right.$ $\left.0) \rightarrow\left(\left(x^{-} \rightarrow x\right) \rightarrow x\right)\right)=f(1)$. So $f\left(\left(x^{-} \rightarrow x\right) \rightarrow\right.$ $x)=f(1)$.

$(3) \Rightarrow(2)$. let $f$ be a fuzzy filter satisfying the condition and let $f((x \rightarrow y) \rightarrow x)=t$, then $(x \rightarrow$ $y) \rightarrow x \leqslant x^{-} \rightarrow x \in f_{t}$. And $f\left(\left(x^{-} \rightarrow x\right) \rightarrow x\right)=$ $f(1)$, then $\left(x^{-} \rightarrow x\right) \rightarrow x \in f_{t}$, therefore $x \in f_{t}$, so $f(x) \geqslant f((x \rightarrow y) \rightarrow x)$. The inverse inequality is obvious.

Corollary 28. Each fuzzy positive implicative filter of $A$ is equivalent to a fuzzy Boolean filter.

Theorem 29. Every fuzzy positive implicative filter of $A$ is a fuzzy fantastic filter of $A$.

Proof. Suppose $f$ is a fuzzy positive implicative filter and for any $x, y \in A$, let $f(y \rightarrow x)=t$, then $y \rightarrow x \in f_{t}$. We have $x \leqslant((x \rightarrow y) \rightarrow y) \rightarrow x$, thus $(((x \rightarrow y) \rightarrow y) \rightarrow x) \rightarrow y \leqslant x \rightarrow y$. Further, $((((x \rightarrow y) \rightarrow y) \rightarrow x) \rightarrow y) \rightarrow(((x \rightarrow y) \rightarrow y) \rightarrow$ $x) \geqslant(x \rightarrow y) \rightarrow(((x \rightarrow y) \rightarrow y) \rightarrow x)=((x \rightarrow y) \rightarrow$ $y) \rightarrow((x \rightarrow y) \rightarrow x) \geqslant y \rightarrow x$. Since $y \rightarrow x \in f_{t}$, hence also $((((x \rightarrow y) \rightarrow y) \rightarrow x) \rightarrow y) \rightarrow(((x \rightarrow y) \rightarrow$ $y) \rightarrow x) \in f_{t}$. Thus we get $((x \rightarrow y) \rightarrow y) \rightarrow x \in f_{t}$, and hence $f$ is a fuzzy fantastic filter.

Corollary 30. Let $f$ be a fuzzy implicative filter of a $B L$-algebra A. Then $f$ is a fuzzy normal filter if only iff is a fuzzy positive implicative filter of $A$.

Corollary 31. Let $f$ be a fuzzy positive implicative filter of A. Then $f$ is a fuzzy normal filter.

Theorem 32. Every fuzzy normal and implicative filter of a BL-algebra is a fuzzy positive implicative filter.

Proof. Suppose $f$ is a fuzzy normal and implicative filter of a $B L$-algebra $A$, For any $x, y, z \in A$, let $f(x) \wedge f(x \rightarrow((y \rightarrow z) \rightarrow y))=t$, then $x \in f_{t}$ and $x \rightarrow((y \rightarrow z) \rightarrow y) \in f_{t}$, then $(y \rightarrow z) \rightarrow y \in f_{t}$, and $(y \rightarrow z) \rightarrow y \leqslant(y \rightarrow z) \rightarrow((y \rightarrow z) \rightarrow z)$, hence $(y \rightarrow z) \rightarrow((y \rightarrow z) \rightarrow z) \in f_{t}$. Since $f_{t}$ is an implicative filter, we can get $(y \rightarrow z) \rightarrow z \in f_{t}$. Then we get $(z \rightarrow y) \rightarrow y \in f_{t}$. From $(y \rightarrow z) \rightarrow y \leqslant z \rightarrow y$, we get $z \rightarrow y \in f_{t}$, combined with $(z \rightarrow y) \rightarrow y \in f_{t}$, then $y \in f_{t}$. So $f_{t}$ is a positive implicative filter of $A$, and thus $f$ is a fuzzy positive implicative filter of $A$.

Corollary 33. Every fuzzy implicative filter satisfying $f((y \rightarrow x) \rightarrow x)=f((x \rightarrow y) \rightarrow y)$ for all $x, y \in A$ of a BL-algebra $A$ is a fuzzy positive implicative filter.

We can get the following theorem.

Theorem 34. Every fuzzy normal and implicative filter of a BL-algebra is a fuzzy fantastic filter.

Proof. Suppose $f(y \rightarrow x)=t$, then $y \rightarrow x \in f_{t}$. We have $x \leqslant((x \rightarrow y) \rightarrow y) \rightarrow x$, thus $(((x \rightarrow y) \rightarrow y) \rightarrow$ $x) \rightarrow y \leqslant x \rightarrow y$. Further, $((((x \rightarrow y) \rightarrow y) \rightarrow x) \rightarrow$ $y) \rightarrow(((x \rightarrow y) \rightarrow y) \rightarrow x) \geqslant(x \rightarrow y) \rightarrow(((x \rightarrow y) \rightarrow$ $y) \rightarrow x)=((x \rightarrow y) \rightarrow y) \rightarrow((x \rightarrow y) \rightarrow x) \geqslant y \rightarrow x$. Since $y \rightarrow x \in f_{t}$, hence also $((((x \rightarrow y) \rightarrow y) \rightarrow$ $x) \rightarrow y) \rightarrow(((x \rightarrow y) \rightarrow y) \rightarrow x) \in f_{t}$. So we get $((x \rightarrow y) \rightarrow y) \rightarrow x \in f_{t}$, and hence $f$ is a fuzzy fantastic filter.

Theorem 35. Every fuzzy normal and obstinate filter of a BL-algebra is a fuzzy fantastic filter.

Proof. Suppose $f$ is a fuzzy normal and obstinate filter of a $B L$-algebra $A$, then $f\left(x^{--}\right)=f(x)$. If $f\left(x^{--}\right)=f(x)=f(1)$, then $f\left(x^{--} \rightarrow x\right) \geqslant f(x)=$ $f(1)$, thus $f\left(x^{--} \rightarrow x\right)=f(1)$. Further, if $f\left(x^{--}\right)=$ $f(x) \neq f(1)$, since $f$ is a fuzzy obstinate filter, then $f\left(x^{--} \rightarrow x\right)=f(1)$, and hence $f$ is a fuzzy fantastic filter.

By Theorem 10, we have

Theorem 36. Every fuzzy normal and ultra filter of a BL-algebra is a fuzzy fantastic filter.

By Theorem 34, Theorem 35, Theorem 36 and Corollary 13, Corollary 25, we can give answers to the first open problem presented in $\left[{ }^{6}\right]$ respectively.

Theorem 37. (1)Every normal filter of an $M V$ algebra is equivalent to a fantastic filter.

(2)Every normal and implicative filter of a $B L$ algebra is a fantastic filter.

(3)Every normal and obstinate filter of a $B L$ algebra is a fantastic filter.

(4)Every normal and ultra filter of a BL-algebra is a fantastic filter. 
For the second open problem presented in $\left[{ }^{6}\right]$, since the extension property for a fantastic filter holds $\left[{ }^{22}\right]$, and further by the above theorems, we have the following theorem.

Theorem 38. (Extension property for a normal filter) Under one of the following conditions extension property for normal filter holds

(1)If $A$ is an MV-algebra.

(2)F is a normal and implicative filter of $A$.

(3) $F$ is a normal and obstinate filter of $A$.

(4) $F$ is a normal and ultra filter of $A$.

\section{Conclusion}

By studying the equivalent condition of fantastic filter, we reveal the relation between fantastic filter and normal filter of $B L$-algebras and solve two open problems regarding the relationship between these two filters of $B L$-algebras. In the future work, we will extend the corresponding filter theory to different algebraic structures, and study the fuzzy congruence relations induced by the fuzzy filters.

\section{Acknowledgments}

This work is partially supported by the National Natural Science Foundation of China (Grant No. 60875034, 61175055); Sichuan Key Technology Research and Development Program of China (Grant No. 2011FZ0051); Wireless Administration of Ministry of Industry and Information Technology of China ([2011]146); the Natural Science foundation of Shaanxi Province (Grant No. 2012JQ1023), China Postdoctoral Science Foundation funded project (Grant No. 2013M540716) and doctor initial fund of Xi' an Shiyou University of China (Grant No. 2011BS017).

\section{References}

1. P. Hájek, "Metamathematics of fuzzy logic," Kluwer, Dordrecht(1998).

2. E. Turunen, "Mathematics Behind Fuzzy Logic," Physica-Verlag, Heidelberg(1999).

3. L.Z. Liu and K.T. Li, "Fuzzy filters of BL-algebras," Information Sciences 173, 141-154 (2005).
4. X.H. Zhang and W.H. Li, "On pseudo- $B L$ algebras and $B C C$-algebras," Soft Computing 10, 941-952(2006).

5. L.Z. Liu and K.T. Li, "Fuzzy Boolean and positive implicative filters of BL-algebras," Fuzzy Sets and Systems 152, 333-348(2005).

6. A.B. Saeid and S. Motamed, "Normal Filters in BLAlgebras," World Applied Sciences Journal 7, 7076(2009).

7. B.V. Gasse, G. Deschrijver, C. Cornelis and E.E. Kerre, "Filters of residuated lattices and triangle algebras," Information Sciences 180, 3006-3020 (2010).

8. J. Mertanen and E. Turunen, "States on semi-divisible generalized residuated lattices reduce to states on $M V$ algebras," Fuzzy Sets and Systems 159, 3051-3064 (2008).

9. L.C. Ciungu, "Algebras on subintervals of pseudohoops," Fuzzy Sets and Systems 160, 10991113(2009).

10. X.H. Zhang and X.S. Fan, "Pseudo- $B L$ algebras and pseudo-effect algebras," Fuzzy sets and systems 159(1), 95-106(2008).

11. O. Xi, "Fuzzy BCK-algebras," Math. Japonica 36, 935-942(1991).

12. Y. Xu and K.Y. Qin, "On filters of lattice implication algebras," Journal of Fuzzy Mathematics 1, 251-260 (1993).

13. Y.B. Jun, "Fuzzy positive implicative and fuzzy associative filters of lattice implication algebras," Fuzzy Sets and Systems 121, 353-357 (2001).

14. Y.B. Jun and S.Z. Song, "On fuzzy implicative filters of lattice implication algebras," Journal of Fuzzy Mathematics 10, 893-900 (2002).

15. X.H. Zhang, Y.B. Jun and M.I. Doh, "On fuzzy filters and fuzzy ideals of BL-algebras," Fuzzy Systems and Mathematics 20, 8-20 (2006).

16. W. Wang and X.L. Xin, "On fuzzy filters of pseudo BL-algebras," Fuzzy Sets and Systems 162, 27-38 (2011).

17. J.M. Zhan, W.A. Dudek and Y.B. Jun, "Interval valued $(\in, \in \vee q)$-fuzzy filters of pseudo BL-algebras," Soft Computing 13, 13-21 (2009).

18. X.L. Ma, J.M. Zhan and K. P. Shum, "Interval valued $(\in, \in \vee q)$-fuzzy filters of MTL-algebras," Journal of Mathematical Research \& Exposition 30, 265-276 (2010).

19. Y.B. Jun, Y. Xu and X.H. Zhang, "Fuzzy filters of MTL-algebras," Information Sciences 175, 120-138 (2005).

20. G. Georgescu, "Bosbach states on fuzzy structures," Soft Computing 8, 217-230 (2004).

21. X.H. Zhang, "Fuzzy logic and its algebric analysis," Science Press, Beijing(2008)

22. J. Rachuånek and D. Šalounová, "Classes of filters in generalizations of commutative fuzzy structures," 48 , 93-107 (2009). 